\title{
Recent developments in track reconstruction and hadron identi- fication at MPD
}

\author{
A. Mudrokh ${ }^{1}$ and $A$. Zinchenko ${ }^{1}$ \\ ${ }^{1}$ Joint Institute for Nuclear Research, Dubna, Russia
}

\begin{abstract}
A Monte Carlo simulation of real detector effects with as many details as possible has been carried out instead of a simplified Geant point smearing approach during the study of the detector performance. Some results of realistic simulation of the MPD TPC (Time Projection Chamber) including digitization in central Au+Au collisions have been obtained. Particle identification (PID) has been tuned to account for modifications in the track reconstruction. Some results on hadron identification in the TPC and TOF (Time Of Flight) detectors with realistically simulated response have been also obtained.
\end{abstract}

\section{Introduction}

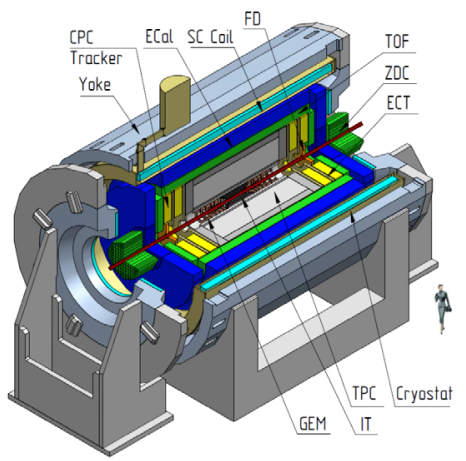

Figure 1. Schematic view of the MPD detector

The MPD detector is a large acceptance spectrometer for heavy-ion collisions study at NICA. The main scientific goal of the NICA/MPD project is to explore the phase diagram of strongly interacting matter in the region of highly compressed and hot baryonic matter [1]. For this purpose an excellent tracking and particle identification (PID) performance over a significant fraction of the final state phase-space are required.

The MPD detector (figure 1) consists of a barrel part and two endcaps located inside the magnetic field. The barrel part is a shell-like set of various detectors surrounding the interaction point and aimed to reconstruct and identify particles in the pseudorapidity region of $|\eta|<1.2$. The endcaps are aimed for precise tracking over pseudorapidity range $1.2<|\eta|<2$. 
The Time Projection Chamber (TPC) is the main MPD tracking detector. It covers the barrel and endcap parts of MPD: its geometrical acceptance limit $|\eta|=2$. TPC is responsible for particle momentum measurements, vertex determination, two track separation, $d E / d x$ measurements and particle identification.

Charged hadrons can be identified in MPD by the TPC and TOF detectors. The mass of the particle is used as an identification parameter, which can be obtained by combining the measurements of its time of flight (from TOF) and its momentum (from TPC).

A new approach based on the full simulation of the detector physics and response is required in order to get realistic estimates of the MPD performance instead of a simplified approach at the smeared hit production level. Most of the physics simulation results obtained so far have been produced for the midrapidity region $|\eta|<1$. An extension of the MPD tracking (and PID) capability up to $|\eta|<2$ would allow us, for example, to increase the proton yield in the event by $50 \%$ which is important in the search for the critical end point.

In this paper only the first results of the TPC tracking and TPC+TOF hadron identification performance studies are presented based on the realistic detector response simulation and respectively tuned cluster, hit, track finding and particle identification procedures.

\section{Realistic simulation of MPD TPC}

Data processing model during the MPD TPC simulation consists of the following steps: event generation, particle transport, TPC response simulation, cluster (hit) finding, tracking and $d E / d x$ reconstruction and physics analysis.

The UrQMD event generator is used as an input for simulation in this study. The transport of particles through the detector is handled by the Geant 3 package.

The following processes are considered in the TPC microsimulation procedure: primary ionization simulation, drift and diffusion of ionization electrons, gas gain fluctuations, pad response, electronics shaping and signal digitization.
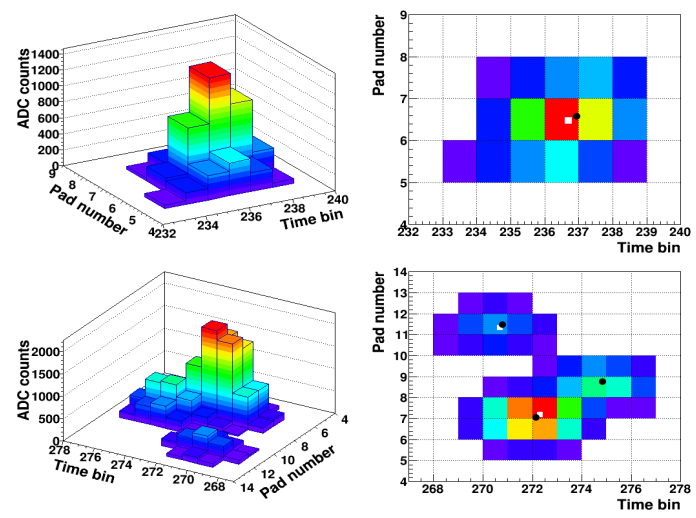

Figure 2. 3D and 2D views of single and triple track clusters

Ionization losses are simulated by Geant in the TPC volume along the trajectory of a charged track. They are converted into the number of electrons which are transported in the uniform electric field toward the anode wires. Both transverse and longitudinal diffusion are considered during the transport process. The charge collected on the sense wire plane is affected by the electron gas multiplication process described by the Polya distribution. The final charge is distributed over readout pads in accordance to the pad response function, which had been determined from a study of a TPC prototype. 
The response of the readout electronics (i.e. shaping preamplifier with $180 \mathrm{~ns}$ FWHM shaping time) is folded with the electron drift time distribution. Finally, all the pad signals are digitized in ADCs with the 12-bit accuracy.

The cluster (hit) finding procedure includes the following steps: finding preclusters, finding separated local maxima using the "peak-and-valley" approach, reconstruction of the coordinates of the found local maxima via center-of-gravities and edge effects corrections.

The "peak-and-valley" approach consists in finding local maxima ("peaks") in the charge $v s$ time bin $v s$ pad number domain diagram (figure 2) separated by sufficiently deep "valleys". The obtained results are shown in figure 3 where the position resolution along the pad row (transverse direction) is plotted as a function of the pad row crossing angle. The spatial resolution along this direction degrades faster in the outer part of TPC where the pad height is larger.

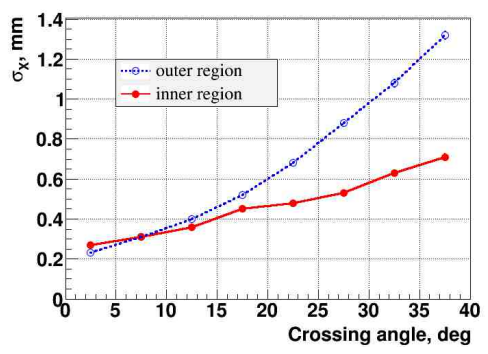

Figure 3. Position resolution along the pad rows for tracks with $p_{t}>0.1 \mathrm{GeV} / \mathrm{c}$ and dip angle below $45 \mathrm{deg}$

Figure 4 demonstrates 2-hit separation capability of the MPD TPC along the pad row direction. The efficiency weakly depends on whether hit is registered in the inner or outer part of the TPC.

The track reconstruction method is based on the Kalman filtering technique. In figure 4 the efficiency of track reconstruction is plotted as a function of transverse momentum for primary particles. The comparison of the realistic simulation with the one obtained with the simplified method, i.e. with Geant points smeared in the transverse direction, shows us the difference between two methods. The observed drop in the TPC tracking efficiency at transverse momenta $p_{t}<200 \mathrm{MeV} / \mathrm{c}$ is presumably due to a larger crossing angle of low- $p_{t}$ tracks and a higher probability to cross inefficient regions at sector boundaries.
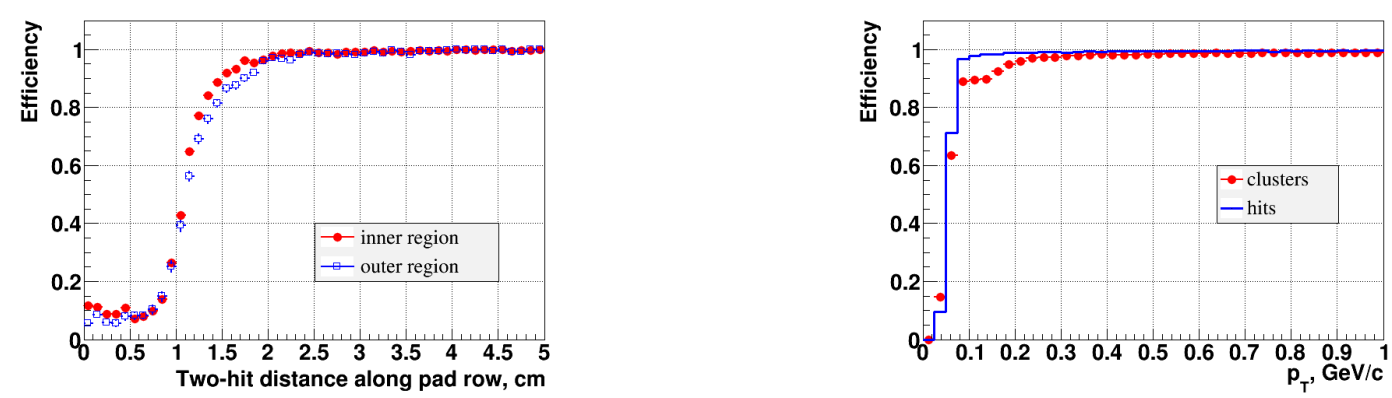

Figure 4. Two-hit resolution along the pad row direction (on the left) and track reconstruction efficiency as a function of $p_{t}$ for primary particles (on the right)

$d E / d x$ measurements are provided by the TPC up to its pseudorapidity acceptance limit (figure 5, left). A truncated mean value is computed for charges of the hits assigned to each track with the truncation level being chosen to obtain the mean energy loss equal to the most probable value (figure 5, right). 


\section{Particle identification (PID) in MPD detector}

One of the main requirements to the MPD experiment to be successful is to provide efficient particle identification. For instance, hadrons $\left(p^{ \pm}, \pi^{ \pm}\right.$and $\left.K^{ \pm}\right)$should be identified over a momentum range from $100 \mathrm{MeV} / \mathrm{c}$ to $3 \mathrm{GeV} / \mathrm{c}$. To ensure that, the energy deposit (from TPC) and mass of particle (from TPC and TOF) are used for reconstructed tracks to get partial (TPC) or combined (TPC+TOF) probabilities of being a particle of a certain specie as explained below. As it has been already mentioned, the detailed microsimulation of the TPC required additional PID tuning which was done on the simulated sample of central (impact parameter $b=0-3 \mathrm{fm}$ ) Au + Au collisions at $\sqrt{s}=6.5 \mathrm{GeV} / \mathrm{c}$ from the UrQMD (ver. 3.4) event generator.

The general idea of particle identification can be illustrated by the definition of the partial probability from the TPC. Charged particles can be identified by the value of their truncated mean energy deposit $d E / d x$ plotted as a function of the full momentum (figure 5, left). For each small interval of momentum $(50 \mathrm{MeV} / \mathrm{c})$ we can produce a normalized energy deposit distribution which is a $d E / d x$ profile for all considered particles in units of the mean pion $d E / d x$ expected for this momentum. As it can be seen from the right plot of figure 5, the normalized energy deposit distribution is a set of (almost) normal distributions, and the main part of the PID task is to parameterize the mutual arrangement of these normal distributions in the whole momentum interval, i.e. to determine the expected mean values, widths and amplitudes of the distributions. Given the PID parameterizations exist, for a reconstructed track with momentum $p$ and energy deposit $d E / d x$ we can obtain a vector of probabilities to belong to each of the particle species.

Currently, such parameterizations have been produced for TPC and TOF identification, but the set of used detectors can be expanded in future.
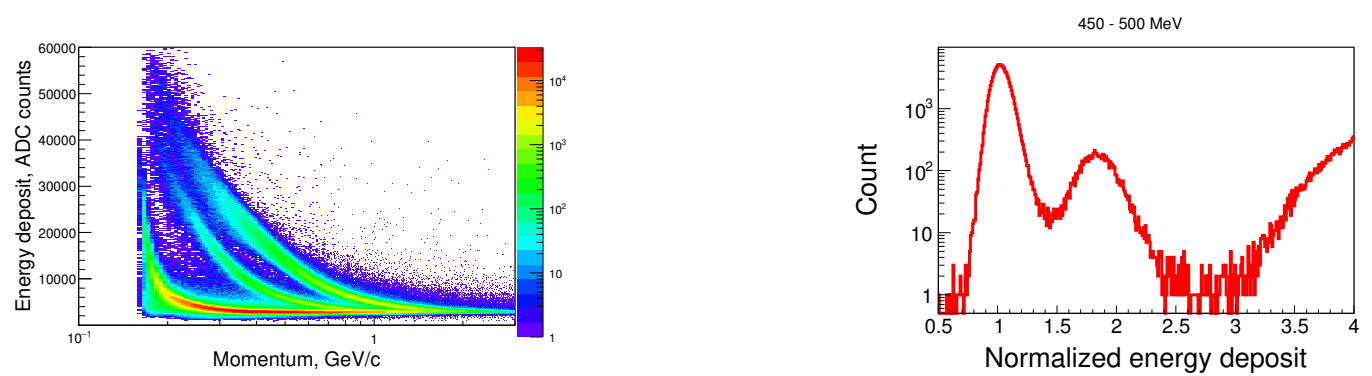

Figure 5. $d E / d x$ reconstruction: energy deposit in ADC counts $v s$ momentum (on the left); projection of energy deposit for particles with $0.45-0.50 \mathrm{GeV} / \mathrm{c}$ momentum (on the right)

According to the general idea described above, hadron identification in MPD can be achieved using the ionization energy loss $(d E / d x)$ from the TPC. The mean $d E / d x$ coincides with the most probable energy deposit after suppressing Landau tail using the truncation method. According to this algorithm, the projection of energy loss at a fixed momentum has a Gaussian form, which is completely defined by three parameters. The mean value of energy loss as a function of full momentum can be described by the Bethe-Bloch function. PID uses simplified Bethe-Bloch function with 5 parameters:

$$
-\frac{d E}{d x}=a_{0}\left\{\frac{p}{\sqrt{p^{2}+m^{2}}}\right\}^{-a_{3}}\left\{a_{1}-{\frac{p}{\sqrt{p^{2}+m^{2}}}}^{a_{3}}-\ln \left(a_{2}+\left(\frac{m}{p}\right)^{a_{4}}\right)\right\},
$$

where $\mathrm{p}$ - full momentum, $\mathrm{a}_{i}$ - fit parameters. 
The parameterization was done for primary particles with the number of hits per track $\geq 10$ and $|\eta|<1$. The ratio of the reconstructed mean $d E / d x$ to the mean value expected from the approximation (1) (figure 6) was determined in order to be sure that parameterization has been done correctly. The ratio has the largest deviation from 1 on the edges of momentum spectrum because of two reasons. First, the simplified Bethe-Bloch function does not well describe behavior of energy deposit for low momenta. And second, the data set had low statistics at high momenta. From the plotted ratio one can obtain also the relative $d E / d x$ width, which is about $7 \%$ in the whole momentum interval.
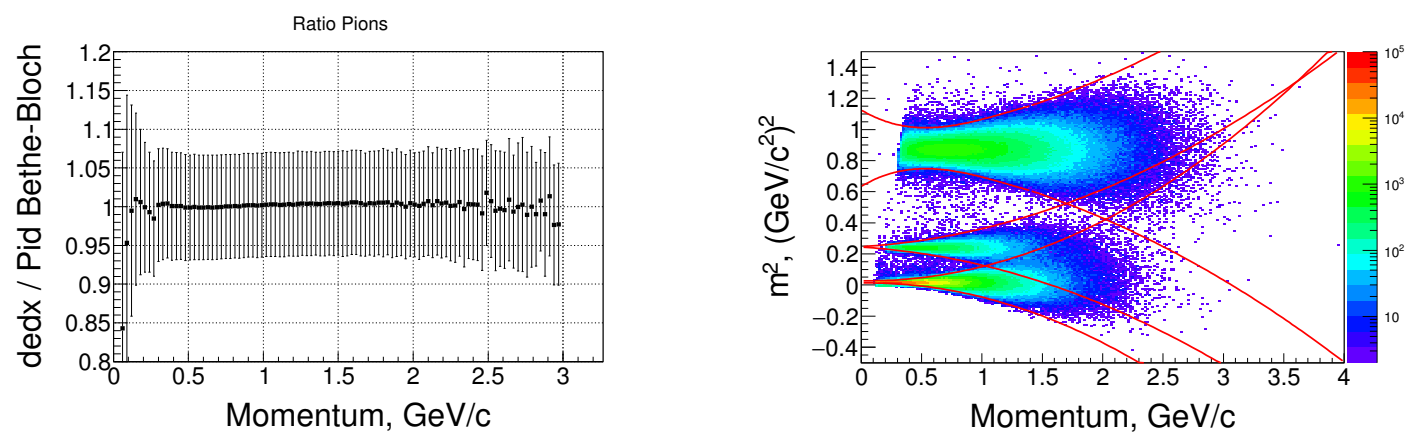

Figure 6. Ratio of the reconstructed mean $d E / d x$ to the value from the approximation 1 (on the left) and dependence of mass-squared on particle momentum (on the right).

Particle identification works much better, if TOF identification is available. It significantly improves PID results for high momenta, where particles cannot be identified by the $d E / d x$ value. TOF identification is based on separation of particles by their reconstructed mass-squared value, which can be calculated from particle momentum and measured time of flight (figure 6 right). Using the procedure, similar to the one described for $d E / d x$-identification, the mass-squared distributions have been produced for different momentum intervals and fitted to the gaussian functions. The obtained widths were fitted by polynomials for every particle specie as it can be seen in figure 6 , where the red lines show $3 \sigma$ mass intervals for different particles.
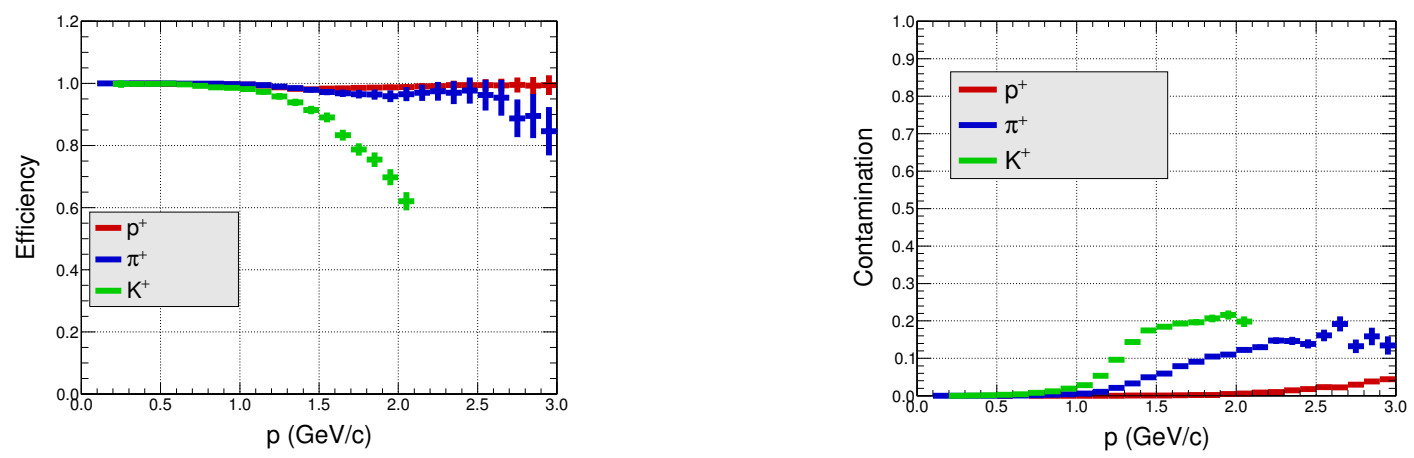

Figure 7. Combined efficiency (on the left) and contamination (on the right) of positive charged particles as a function of full momentum

The PID performance can be characterized by the efficiency and contamination dependencies on the full momentum (figure 7). The combined efficiency and contamination were obtained for positively charged particles which were identified by the PID probability above $70 \%$. As it can be seen, this condition is too strong for kaons, causing their efficiency dropping at high momenta. 


\section{Conclusions}

The MPD TPC cluster finder method based on the detailed microsimulation procedure has been developed. Simulation results have been compared with the ones obtained with the gaussian smearing approach. Further work on the reconstruction algorithm fine tuning is foreseen.

PID parameterizations have been also obtained. Efficient $\pi / K$ separation up to $1.5 \mathrm{GeV} / \mathrm{c}$ and $\pi / p$ separation up to $3 \mathrm{GeV} / \mathrm{c}$ has been demonstrated.

As a continuation of this work, the extension of the track reconstruction and particle identification up to $|\eta|=2$ is planned.

\section{References}

[1] K.U. Abraamyan et al., Nucl. Instrum. Meth. A 628, 99 (2011) 